\title{
Incidencia de la utilización de herramientas informáticas en el estudio del Límite de una Función Real
}

\section{Impact of the use of computer tools in the study of the Limit of a Real Function}

\author{
Wilson Román ${ }^{\mathrm{a}}$, Norma Barreno ${ }^{\mathrm{a}}$, Franklin Coronel $^{\mathrm{b}}$ \\ ${ }^{a}$ Departamento de Ciencias Exactas, Universidad de las Fuerzas Armadas-ESPE \\ ${ }^{\text {b }}$ Facultad de Ingeniería Electrónica /Escuela de Telecomunicaciones, Escuela Superior Politécnica de Chimborazo
}

Correspondencia Autores: wmroman@espe.edu.ec,npbarreno@espe.edu.ec, fcoronel@espoch.edu.ec

Recibido: junio 2018, Publicado: diciembre 2018

\begin{abstract}
Resumen - La investigación aborda el problema de reprobación y deserción de los estudiantes en la asignatura de cálculo diferencial; en la fase exploratoria del proyecto se identifica el tema de límite de una función real como una de las definiciones abstractas y difíciles de comprender por parte del estudiante; para abordar el presente problema se considera analizar la incidencia de la utilización de herramientas informáticas como un recurso didáctico que permita lograr aprendizajes significativos mejorando el rendimiento académico de los estudiantes, se considera dos grupos de estudiantes identificado como grupo de control y grupo de experimentación, se aplica el cuestionario CHAEA para identificar el estilo de aprendizaje predominante de cada estudiante, con estos resultados se diseñan e implementan diferentes metodologías de evaluación académica y con la utilización de rubricas se definen los criterios de evaluación en función del estilo de aprendizaje, el objetivo es medir la incidencia de las aplicaciones informáticas en el rendimiento académico y el aprendizaje a largo tiempo, para lo cual se implementan tres fases de análisis denominados, pretest, test y postest, en cada uno de ellos se implementan análisis estadísticos con un nivel de significancia del 0.05 para los contrastes de promedios, el análisis de varianza y la correlación entre los estilos de aprendizaje, en la fase del test se obtiene un p-valor de 0.10 para la prueba estadística $\mathrm{F}$, cuya regla de decisión indica que no existe una diferencia significativa en el rendimiento académico de los estudiantes que utilizaron las aplicaciones informáticas con respecto al grupo que no utilizo; pero, en el postest al contrastar las medias entre los grupos se tiene un p-valor de 0.000 en el estadístico t, es decir, se verifica que el grupo experimental demuestra un aprendizaje duradero con respecto al grupo de control, por lo tanto, es recomendable la utilización de herramientas informáticas como una estrategia didáctica en el aula de clases.
\end{abstract}

Palabras Claves - Aprendizaje significativo, estilos de aprendizaje, metodologías didácticas, herramientas informáticas.

\footnotetext{
Abstract - The research addresses the problem of students' failure and dropout in the subject of differential calculus; in the exploratory phase of the project, the theme of the limit of a real function is identified as one of the abstract and difficult definitions to understand by the student; To take into account to take into account to take into account to take into account to take into account to take into account to obtain information. applies the CHAEA questionnaire to identify the predominant learning style of each student, with these results are designed and implemented different academic assessment methodologies and the use of rubrics are used to evaluate the role of learning style, the objective is to measure the incidence of computer applications in academic performance and long-term learning, for what is implemented in the analysis phases called, pretest, test and posttest, in each of them is implemented a
}

statistical analysis with a level of significance of 0.05 for the contrasts of averages, the analysis of the variance and the correlation between the learning styles, the phase of the test we obtain a value of $\mathrm{p} 0.10$ for the statistical test $\mathrm{F}$, the decision rule indicates that there is no difference significant in the performance the content of the students that uses the computer applications with respect to the group that does not use lists; but, in the posttest, when contrasting the means between the groups, a value of 0.000 is taken into account in the statistic, that is, it is verified that the experimental group demonstrates a lasting learning with respect to the control group, therefore, it is the use of computer tools as a didactic strategy in the classroom.

Keywords - Significant learning, learning styles, didactic methodologies, computer tools.

\section{INTRODUCCIÓN}

En la asignatura de Cálculo Diferencial e Integral que se imparte en los primeros niveles de educación universitarias se presenta un problema generado en el proceso de enseñanza-aprendizaje de la definición del límite de una función real. Para [1] el estudio del límite de una función real representa uno de los grandes inconvenientes que enfrentan los estudiantes al iniciar sus estudios superiores, en el trabajo realizado por [2] se analiza el rendimiento académico y el abandono universitario el mismo que ocurre generalmente en los primeros niveles de la educación superior, en [3] se menciona como uno de los factores relacionados con la deserción estudiantil. Por lo tanto, la investigación se orienta en diseñar, implementar y analizar la incidencia de la utilización de aplicaciones informáticas, con la finalidad de mejorar el rendimiento académico y lograr aprendizajes significativos, que según [4] promueve la implementación de modelos educativos centrados en el estudiante. Además, para [5] es necesario que el docente este altamente capacitado para asumir los nuevos retos de la formación académica de un estudiante.

La globalización del conocimiento impone retos y desafíos a las instituciones de educación superior, el desarrollo tecnológico exige la formación de ingenieros altamente competitivos con una sólida formación matemática, lo que obliga a replantear contenidos y metodologías de enseñanza, para conseguir estudiantes motivados, creativos e innovadores; integrando el saber, el 
saber hacer y el saber ser, características descritas en [6]. En el trabajo realizado por [7] se plantea: ¿Qué educación matemática puede preparar a los estudiantes para la sociedad del futuro? Revisando [8] se determina los lineamientos sobre competencias matemáticas de un estudiante enmarcados en la habilidad para utilizar y relacionar formas de expresión simbólicas, razonamientos matemáticos, resolución de problemas relacionados con la vida cotidiana y el perfil profesional de la carrera, permitiendo ampliar el conocimiento sobre aspectos cuantitativos y espaciales de la realidad.

La importancia de la matemática en la formación académica de un ingeniero descrita en [9], visualiza la necesidad de un alto componente cognitivo en el análisis matemático fundamentado en el dominio y aplicación de la definición del límite de una función enunciada por Cauchy y descrita en [10], ésta definición constituye la piedra angular para el desarrollo del análisis matemático real, análisis funcional, análisis armónico y análisis complejo; su concepto se aplica en el estudio de las Series y Transformadas de Fourier que tienen un sinnúmero de aplicaciones entre ellas el procesamiento de imágenes, originando nuevas transformadas como las Wavelet, Random y otras. Por tal razón, la trascendencia de la investigación sobre el tema del límite de una función real.

\section{MATERIALES Y MÉTODOS}

La investigación es de tipo causa-efecto, de carácter explicativa porque busca establecer relación entre los factores que intervienen en el problema que se investiga; además, la investigación es de carácter evaluativa porque se implementa la resolución de problemas para verificar la eficiencia y eficacia de la parte cognitiva del tema; la exposición para medir la fluidez de transmitir lo aprendido; el desarrollo de prácticas, para validar la aplicación de los conceptos y las pruebas de selección múltiple para consolidar el dominio del tema [11].

El enfoque de la investigación es cuantitativo, ya que, se utiliza la recolección de datos para validar la hipótesis en función del análisis estadístico de los registros de rendimiento académico obtenidos entre el grupo de control y el grupo de experimentación. El diseño de la investigación es de tipo cuasi-experimental, no es necesario la determinación de una muestra en la investigación. Para el grupo experimental se aplicó la metodología didáctica basada en la resolución de problemas mediante la utilización de herramientas informáticas y al grupo de control se desarrolló las clases con la metodología didáctica basada en la resolución de problemas y clases magistrales.

\section{A. Análisis del problema}

Para el análisis del problema planteado se utiliza el diagrama causa-efecto descrito en la Fig. 1, como herramienta de análisis que nos permita obtener un cuadro detallado y de fácil visualización de las diversas causas que pueden originar el problema de investigación; se menciona como causa del problema la utilización de cuantificadores en la definición del límite de una función, la necesidad de realizar varias gráficas con la intención de explicar el tema, pero las mismas son representaciones estáticas y no dinámicos que no permiten una interacción entre el estudiante y el objeto de estudio, y como consecuencia se consiguen estudiantes desmotivados, con dificultades de poder aplicar la definición del límite en la solución de ejercicios.

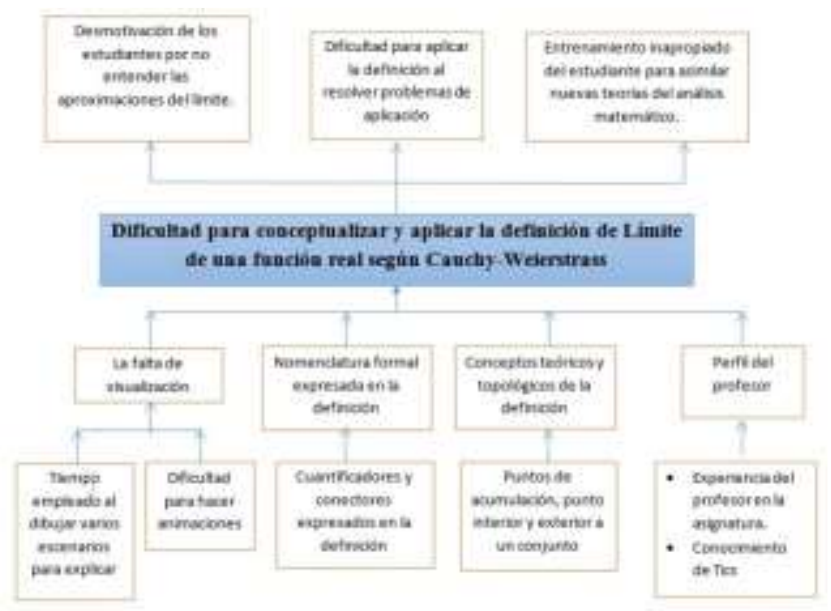

Fig. 1. Diagrama de causas-efectos acerca de la dificultad para conceptualizar y aplicar la definición de Límite a una función real. Fuente: Autores en base al Diagramas Ishikawa

\section{B. Método y estilos de enseñanza-aprendizaje}

Para el desarrollo del trabajo de investigación se considera un solo docente y dos grupos de estudiantes que reciben la asignatura de Cálculo Diferencial e Integral en la carrera de Ingeniería Automotriz de la Universidad de las Fuerzas Armadas-ESPE extensión Latacunga; definiéndoles como grupo de experimentación y grupo de control con 30 estudiantes matriculados en cada curso; las metodologías utilizadas para los dos grupos de investigación son las mismas, siendo: exposición del tema y su campo de aplicación, pruebas de selección múltiple, desarrollo de prácticas experimentales y la resolución de problemas, todos estos instrumentos son evaluados utilizando rúbricas con la definición de cinco conceptos con la misma puntuación y cada concepto tiene una escala de valoración de 4 para sobresaliente, 3 muy bueno, 2 bueno y 1 regular, generando una nota sobre 20 puntos, las diferentes evaluaciones se ejecutan el mismo día para los dos grupos.

En el trabajo de [12] se implementa el cuestionario CHAEA como un instrumento para determinar el estilo de aprendizaje de cada estudiante categorizados como Reflexivo, Pragmático, Teórico y Activo, [13] el cuestionario consta de ochenta ítems de respuesta dicotómica, la distribución de las preguntas en cada estilo de aprendizaje son de forma aleatoria, la puntuación total obtenida por cada estudiante en cada grupo de veinte ítems indica el nivel que éste alcanza en cada uno de los cuatro estilos. De esta forma, se obtienen los datos para valorar las preferencias y obtener el perfil de aprendizaje [14].

En la tabla 1, se presenta la reformulación de los estilos de aprendizaje propuestos por Kolb y Honey-Mundford, descritos en [15]; además, en [16] se presenta algunos indicadores de idoneidad didáctica sobre procesos de enseñanza y aprendizaje de la matemática, lo que permite dar 
las siguientes características para los estudiantes que son parte de la investigación.

\begin{tabular}{|c|c|}
\hline ESTILO & CARACTERÍSTICA \\
\hline $\begin{array}{l}\text { Activo- } \\
\text { improvisador }\end{array}$ & $\begin{array}{l}\text { El cometer errores no representa causa de } \\
\text { frustración, son improvisadores y espontáneos. } \\
\text { Retienen mejor la información cuando aplican el } \\
\text { conocimiento en el desarrolla de guías de estudio, } \\
\text { talleres y otros; se adapta con facilidad a espacios } \\
\text { de debates y discusión académica. }\end{array}$ \\
\hline $\begin{array}{l}\text { Teórico- } \\
\text { metódico } \\
\text { objetivo }\end{array}$ & $\begin{array}{l}\text { Son estudiantes objetivos, con un profundo sentido } \\
\text { crítico, metódico y disciplinado, que abordan los } \\
\text { problemas desde un punto de vista lógico. } \\
\text { Prefieren las actividades estructuradas que les } \\
\text { permitan comprender sistemas complejos. } \\
\text { Prefieren las clases magistrales. }\end{array}$ \\
\hline $\begin{array}{l}\text { Pragmático- } \\
\text { realista }\end{array}$ & $\begin{array}{l}\text { Son estudiantes que prefieren las abstracciones, } \\
\text { para resolver los problemas al analizar diferentes } \\
\text { posibilidades y relaciones fundamentadas en la } \\
\text { innovación bajo un enfoque realista, directo, eficaz } \\
\text { y práctico, prefieren planificar las acciones de } \\
\text { manera que puedan ver relación entre el asunto } \\
\text { tratado y su aplicación. }\end{array}$ \\
\hline $\begin{array}{l}\text { Reflexivo- } \\
\text { analítico }\end{array}$ & $\begin{array}{l}\text { Son estudiantes que prefieren analizar } \\
\text { detenidamente el objeto de estudio, generalmente } \\
\text { prefieren trabajar solos. Prefieren la elaboración de } \\
\text { mapas conceptuales, diagramas de flujo, árboles } \\
\text { de problemas. }\end{array}$ \\
\hline
\end{tabular}

En [17] se manifiesta que existe una estrecha relación entre el estilo de aprendizaje, la estrategia didáctica utilizada y el aspecto motivador de la transferencia del conocimiento, en este contexto la presente investigación propone como estrategia la utilización de herramientas informáticas basadas en la utilización del software con la intención de crear ambientes altamente motivados en los procesos de enseñanza-aprendizaje del límite de una función real, además, se incorpora la técnica didáctica de resolución de problemas bajo el enfoque educativo por competencias, que según [18] indica que la incidencia debe ser reflejado en el resultado de la evaluación del estudiante.

En el trabajo realizado por [19] se determina una escala para identificar el estilo de aprendizaje predominante descrita en la tabla 1, en [20] se presentan las puntuaciones correspondientes a cada estilo de aprendizaje y su nivel de categorización; además, en la tabla 2, se determina el estilo de aprendizaje predominante en cada uno de los estudios que son parte de la investigación.

Tabla 2: Preferencia de estilo de aprendizaje de los estudiantes [14]

\begin{tabular}{|c|c|c|c|c|c|}
\cline { 2 - 6 } \multicolumn{1}{c|}{} & $\begin{array}{c}\text { Muy } \\
\text { baja } \\
(\mathbf{1 0 \%})\end{array}$ & $\begin{array}{c}\text { Baja } \\
\mathbf{( 2 0 \% )}\end{array}$ & $\begin{array}{c}\text { Moderada } \\
\mathbf{( 4 0 \% )}\end{array}$ & $\begin{array}{c}\text { Alta } \\
\mathbf{( 2 0 \% )}\end{array}$ & $\begin{array}{c}\text { Muy alta } \\
(\mathbf{1 0 \%})\end{array}$ \\
\hline Activo & $0-6$ & $7-8$ & $9-12$ & $13-14$ & $15-20$ \\
\hline Reflexivo & $0-10$ & $11-13$ & $14-17$ & $18-19$ & 20 \\
\hline Teórico & $0-6$ & $7-9$ & $10-13$ & $14-15$ & $16-20$ \\
\hline Pragmático & $0-8$ & $9-10$ & $11-13$ & $14-15$ & $16-20$ \\
\hline
\end{tabular}

La investigación presenta dos grupos de estudiantes enmarcados en Teórico-Reflexivo caracterizado por identificarse con situaciones estructuradas de forma conceptuales y teóricas, capacidad para cuestionar con argumentos metódicos y lógicos, observa, escucha y reflexiona para poder ejecutar actividades que le conduzcan a logros de aprendizajes; el Activo-Pragmático caracterizado por competir en equipo, en busca de nuevas experiencias, para experimentar y practicar técnicas con asesoramiento del docente o de algún experto, resuelve problemas en función de un método predominado, le apasiona realizar diferentes simulaciones en la búsqueda de soluciones a problemas de la vida real.

\section{Herramienta informática}

En la Fig. 2 se describen las fases para el desarrollo de las aplicaciones informáticas [21], la metodología tiene una fase transversal dada por la motivación del estudiante, la parte cognitiva del tema centrado en el conocimiento y los aspectos didácticos-pedagógicos del docente, de forma vertical se genera el vínculo entre el estudiante y la utilización de las aplicaciones informáticas que según [22] conduce al logro de aprendizajes colaborativos y significativos que se enmarca en el trabajo realizado por [23] sobre la teoría de los campos conceptuales que facilitan aprendizajes significativos.

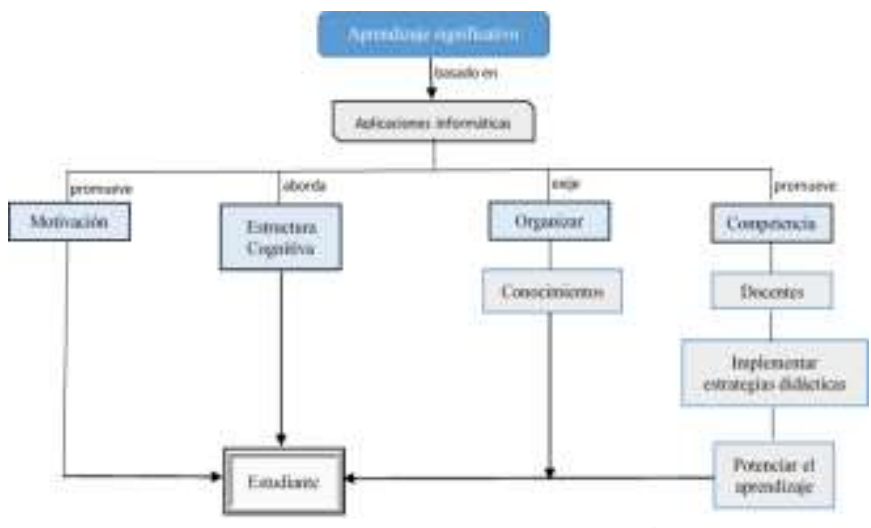

Fig. 2. Fases del diseño de las aplicaciones informáticas Fuente: Autores en base a un diagramas de flujo

Tabla 3: Análisis de las herramientas informáticas según las teorías del aprendizaje

\begin{tabular}{|c|c|c|}
\hline $\begin{array}{c}\text { TIPOS DE } \\
\text { PROGRAMAS }\end{array}$ & $\begin{array}{l}\text { TEORÍAS DEL } \\
\text { APRENDIZAJE }\end{array}$ & $\begin{array}{c}\text { MODELOS } \\
\text { INSTRUCTIVOS }\end{array}$ \\
\hline $\begin{array}{l}\text { Maxima } \\
\text { Wiris } \\
\text { Scilab }\end{array}$ & $\begin{array}{ll}\text { - } & \text { Conductismo } \\
\text { - } & \text { Cognitivismo }\end{array}$ & $\begin{array}{l}\text { - } \\
\text { en la enseñananza } \\
\text { programada. } \\
\text { Aprendizaje basado } \\
\text { en el } \\
\text { almacenamiento y la } \\
\text { representación de la } \\
\text { información. }\end{array}$ \\
\hline Geogebra & $\begin{array}{ll}\text { - } & \text { Cognitivismo } \\
\text { - } & \text { Constructivismo } \\
& \text { Teorías sociales } \\
\text { del aprendizaje }\end{array}$ & $\begin{array}{l}\text { - Aprendizaje basado } \\
\text { en el } \\
\text { almacenamiento y la } \\
\text { representación de la } \\
\text { información. } \\
\text { Aprendizaje basado } \\
\text { en el descubrimiento. } \\
\text { Aprendizaje } \\
\text { colaborativo. }\end{array}$ \\
\hline
\end{tabular}

Nota: Tabla elaborado por los autores con la intención de evidenciar las características del software libre investigado y su relación con las teorías del aprendizaje considerando el nivel de conocimiento que posee el estudiante en aspectos de programación.

En el tabla 3, se presenta el resumen de la investigación realizada para la selección del software libre, la revisión 
bibliográfica se fundamenta en el trabajo realizado por [6] sobre los efectos de la tecnología educativa en un salón de clases, considerando que es necesario considerar su capacidad y facilidad para acceder a la información, su versatilidad para ingresar datos, disponibilidad de utilitarios para generar escenarios dinámicos de análisis; para [20] la influencia de los estilos de aprendizaje y la metacognición en el rendimiento académico se orientan en los modelos instructivos de la teoría de aprendizajes y [24] presenta los efectos del software libre para lograr una matemática dinámica dada en escenarios interactivo del análisis de las funciones reales.

En [25] se proporciona un instrumento de evaluación para software educativo, de los resultados obtenidos se determina que el software Geogebra se adapta de mejor manera a los objetivos de la investigación, por disponer de herramientas que contribuyen en el desenvolvimiento de adquisición y consolidación del conocimiento, a través de su facilidad para el desarrollo de diferentes prácticas, características formuladas en los trabajos de [1] y [26]; en tanto que el software Maxima y Scilab necesitan de conocimientos previos del estudiante sobre aspectos de programación, y este aspecto podría distraer el objetivo de la investigación.

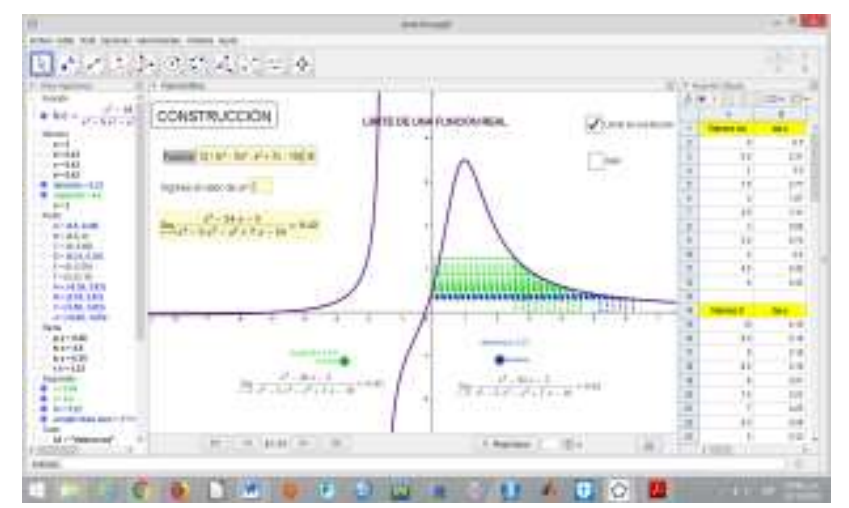

Fig. 3. Visualiza los entornos de evaluación del límite, permite realizar Fuente: Autores animaciones didácticas.

En la Fig. 3. se presenta una de las aplicaciones realizadas utilizando el software Geogebra, que dispone de las Vistas Múltiples de los Objetos Matemáticos en la que se puede observar la Vista Gráfica, Vista Algebraica y la Vista de hoja de cálculo que son los principales escenarios de trabajo del Geogebra. Todo objeto creado en la Vista Gráfica, tiene su representación en la Vista Algebraica y las acciones se registran en el protocolo de construcción; mediante la utilización de las barras de menú y las diferentes herramientas disponibles se realiza el diseño y construcción de las funciones reales, con los deslizadores se simulan el comportamiento de las imágenes de la función de forma gráfica para verificar su tendencia, y mediante la hoja de cálculo se obtiene automáticamente los valores de la función evaluadas en cada punto, de esta forma se le permite al estudiante comprobar el cálculo del límite de una función real, se puede observar y analizar el comportamiento de sus variaciones; para [24] el software Geogebra permite hablar de matemáticas dinámicas porque en su estructura tiene implementado plantillas dinámicas que facilitan el aprendizaje en varios campos del álgebra, la geometría, el análisis matemático y otros campos de la matemática, en [27] se destaca el efecto comparativo de la enseñanza asistida utilizando un ordenador, la misma que motiva la creatividad del estudiante en clases de matemáticas en contra parte de la forma tradicional de transmitir los conocimientos.

\section{Definición de límite de una función real}

Sea $f: A \rightarrow B$ una función real con $A, B \subset \mathbb{R}$, se dice que $f(x)$ tiene límite $L \in \mathbb{R}$ cuando $x$ tiende a $x_{0}$ y se denota con $\lim _{x \rightarrow x_{0}} f(x)=L$ si y solo si

$\forall \varepsilon>0 \quad \exists \delta>0$ tal que $\forall x \in A$ se cumple $\left|x-x_{0}\right|<\delta \Rightarrow$ $|f(x)-L|<\varepsilon$

Por ejemplo, verificar el siguiente límite de una función real.

$$
\lim _{x \rightarrow-\underbrace{}_{x_{0}}} \underbrace{3 x^{2}+x-1}_{f(x)}=\underbrace{1}_{L}
$$

Requiere de un desarrollo analítico que implica la aplicación de la definición de límite incluido el proceso de acotación de funciones reales.

Límites laterales: siempre que se pueda aproximar al punto $x_{0}$ que se encuentra en un entorno $] x_{0}-\delta, x_{0}+\delta[$, se podrá definir los límites laterales por la izquierda y derecha.

Límite lateral por la derecha:

$$
\begin{aligned}
\lim _{x \rightarrow x_{0}} f(x)=L & \Leftrightarrow \forall \varepsilon>0 \exists \delta>0 / x_{0}<x<x_{0}+\delta \\
& \Rightarrow|f(x)-L|<\varepsilon
\end{aligned}
$$

Límite lateral por la izquierda:

$$
\begin{aligned}
\lim _{x \rightarrow x_{0}} f(x)=L & \Leftrightarrow \forall \varepsilon>0 \exists \delta>0 / x_{0}-\delta<x<x_{0} \\
& \Rightarrow|f(x)-L|<\varepsilon
\end{aligned}
$$

Luego, existe el límite de una función real si y solo si, los límites laterales existen y son iguales; es decir,

$$
\exists \lim _{x \rightarrow x_{0}} f(x)=L \leftrightarrow \lim _{x \rightarrow x_{0}} f(x)=\lim _{x \rightarrow x_{0}} f(x)=L
$$

Al resolver analíticamente el ejercicio presentado en la Fig. 3, se tiene el siguiente desarrollo,

$$
\begin{gathered}
\lim _{x \rightarrow 5} \frac{x^{3}-24 x-5}{x^{4}-5 x^{3}-x^{2}+7 x-10} \\
=\frac{5^{3}-24(5)-5}{5^{4}-5(5)^{3}-5^{2}+7(5)-10}=\frac{0}{0}
\end{gathered}
$$

Luego, la forma de indeterminación presente se evita mediante factorización; es decir,

$$
\lim _{x \rightarrow 5} \frac{x^{3}-24 x-5}{x^{4}-5 x^{3}-x^{2}+7 x-10}
$$


$=\lim _{x \rightarrow 5} \frac{(x-5)\left(x^{2}+5 x+1\right)}{(x-5)\left(x^{3}-x+2\right)}=\lim _{x \rightarrow 0} \frac{\left(x^{2}+5 x+1\right)}{\left(x^{3}-x+2\right)}=\frac{51}{122}$

De esta forma se obtiene el resultado del cálculo del límite, pero se deja de lado el análisis de la parte conceptual de límite en el sentido de <<proximidad>>, convirtiendo el cálculo en procesos aburridos y estresantes por conseguir un resultado dado en el texto guía, en tanto que la aplicación informática permite realizar procesos de construcción, visualización y animación del caso de estudio, que según [28] lo presenta como aspectos importantes en la generación de ambientes de aprendizajes dinámicos y reflexivos.

\section{RESULTADOS}

Se considera tres fases de análisis identificadas como el pretest, test y postest; para validar aspectos cognitivos y actitudinales de los estudiantes, el instrumento considerado es la Guía de estudios que contiene una serie de ejercicios a ser desarrollados en función del estilo de aprendizaje del estudiante y la metodología de evaluación implementada a partir de la utilización de rubricas, las mismas que contienen la matriz de los criterios, sus dimensiones y el nivel de desempeño.

En el pretest, se aplica una prueba de diagnóstico para identificar los conocimientos previos de los grupos de estudiantes sobre el tema de funciones reales, en la tabla 4 se presenta las estadísticas descriptivas de los resultados.

Tabla 4

Estadísticos descriptivos de la prueba de diagnóstico en el pretest

\begin{tabular}{|c|c|c|c|c|}
\hline Grupos & N & Media & $\begin{array}{c}\text { Desviación } \\
\text { típ. }\end{array}$ & $\begin{array}{c}\text { Error típ. } \\
\text { de la media }\end{array}$ \\
\hline G_Control & 30 & 16.67 & 1.13 & 0.32 \\
\hline $\begin{array}{c}\text { G_Experiment } \\
\text { ación }\end{array}$ & 30 & 16.31 & 1.73 & 0.21 \\
\hline
\end{tabular}

Se puede observar que el grupo de control tiene una media aritmética de 16.67/20 y el grupo de experimentación de $16.31 / 20$ puntos; es decir, el promedio de los dos grupos son semejantes, para garantizar esta interpretación se implementa la Prueba de Normalidad de Shapiro-Wilk, los resultados se presentan en la tabla 5 donde el p-valor dado por Sig. son mayores que 0.05 , entonces se concluye que los datos corresponden a distribuciones normales. Este hecho nos permite garantizar que no existe mayor diferencia entre los grupos de estudiantes que son parte de la investigación.

Tabla 5

Estadísticos descriptivos de la prueba de diagnóstico en el pretest

\begin{tabular}{|l|c|c|c|}
\hline \multirow{2}{*}{\multicolumn{1}{c}{ Grupos }} & \multicolumn{3}{|c|}{ Shapiro-Wilk } \\
\cline { 2 - 4 } & Estadístico & Gl & Sig. \\
\hline G_Experimentación & 0.889 & 30 & 0.056 \\
\hline G_Control & 0.938 & 30 & 0.081 \\
\hline
\end{tabular}

En la Fig. 4, se presentan las frecuencias de los estilos de aprendizaje de los estudiantes en cada uno de los grupos de investigación obtenidos al contabilizar los resultados del cuestionario CHAEA y categorizados mediante la aplicación de la tabla 1 y la tabla 2, solo como Reflexivo, Pragmático,
Teórico y Activo, se determina que la mayor frecuencia esta dado en los estudiantes con estilo de aprendizaje activo, en tanto que con menor frecuencia se identifica al estilo de aprendizaje reflexivo.

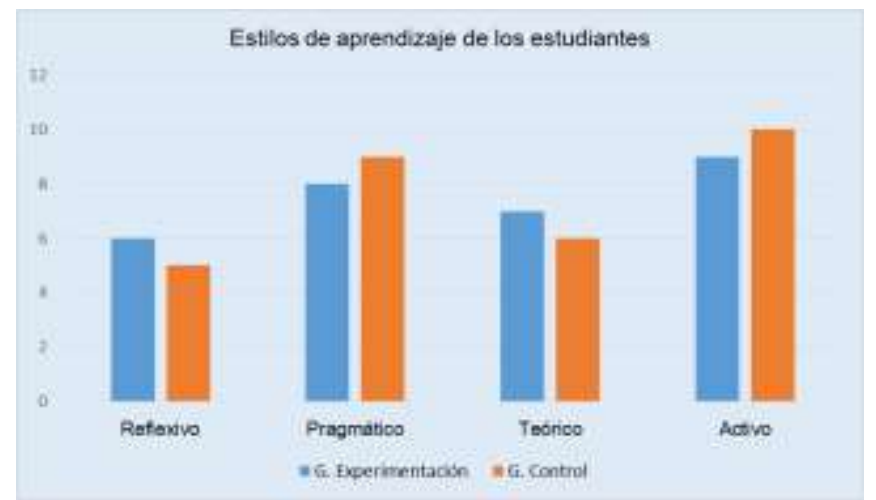

Fig. 4. Frecuencias de los Estilos de aprendizaje de los estudiantes Fuente: Grupos de estudiantes que intervienen en la investigación, resultados del cuestionario CHAEA [14]

En los grupos de estudiantes se identifica comportamientos similares con respecto a los estilos de aprendizaje, el estilo de aprendizaje activo tiene mayor frecuencia, 10 para el grupo de control y 9 para el grupo de experimentación.

Las metodologías de evaluación considerados en el test son exposiciones, pruebas de selección múltiple, desarrollo de aplicaciones informáticas y resolución de problemas, el diseño y elaboración de los instrumentos está sujeto a valoración mediante el coeficiente Alfa de Cronbach el cual es calculado por

$$
\alpha=\frac{n p}{1+p(n-1)}
$$

Donde:

$\alpha=$ es el Coeficiente de Alfa de Cronbach

$\mathrm{n}=$ número de ítems

$\mathrm{p}=$ promedio de correlaciones lineales entre cada uno de

los ítems

$$
\alpha=\frac{10 * 0.8}{1+0.8 *(10-1)}
$$

$\alpha=0.9756$ equivalente a $97.56 \%$

Como el valor calculado $97.56 \%$ es superior a $75 \%$ que requiere la prueba de valoración de los instrumentos de evaluación, entonces se garantiza la aplicabilidad para evaluar a los estudiantes y verificar el cumplimiento de los objetivos de investigación y validación de la hipótesis.

En la Fig. 5, se muestran los resultados descriptivos de los promedios de las calificaciones obtenidos por los estudiantes en cada uno de los parámetros de evaluación, en el desarrollo de las prácticas experimentales que implican la utilización de las aplicaciones informáticas se identifica una amplia diferencia en los promedios entre el grupo de experimentación con 18.3/20 y el grupo de control con $16.4 / 20$ puntos, en la rúbrica utilizada para la evaluación de 
la metodología de Desarrollo de Prácticas Experimentales, la diferencia de puntuación está dada en el concepto de describir el procedimiento para definir y aplicar la definición de límite de una función en la solución de problemas de la vida real.

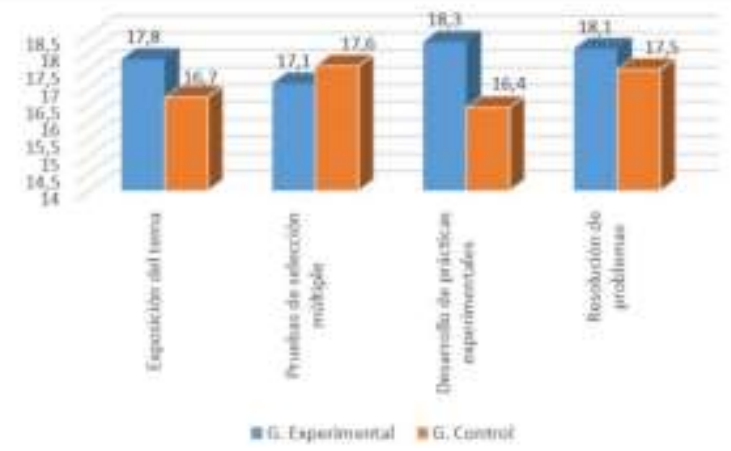

Fig. 5. Comparación del promedio de notas obtenidas tras la aplicación de los diferentes instrumentos de evaluación (por grupos)

Fuente: Evaluaciones de los grupos de estudiantes que intervienen en la investigación

Además, de la Fig. 5, se obtienen los promedio de cada metodología aplicada a los grupos de investigación y con estos valores se obtiene el promedio general de cada curso, siendo el promedio del grupo de experimentación de 17.83/20 y del grupo de control de 17.05/20. Se aplica un análisis de varianza para la verificación de la hipótesis, para los cual se emplea el estadístico F de Fisher, los resultados se presentan en la tabla 6, se tiene que el F crítico es de 5.99 que es mayor que la F calculado con 3.84; por lo tanto, se concluye que no existe diferencia significativa en el rendimientos académico entre las varianzas de los dos grupos, análisis efectuado al $95 \%$ del nivel de confianza.

Tabla 6

Análisis de Varianza entre las calificaciones de los dos grupos

\begin{tabular}{|c|c|c|c|}
\hline $\begin{array}{c}\text { Origen de las } \\
\text { variaciones }\end{array}$ & F & Probabilidad & $\begin{array}{c}\text { Valor crítico } \\
\text { para F }\end{array}$ \\
\hline Entre grupos & 3.84 & 0.10 & 5.99 \\
\hline
\end{tabular}

Los valores del estadístico F y F crítico, permiten tomar la decisión; por lo tanto, las herramientas informáticas implementadas como una estrategia de aprendizaje si aporta en el aprendizaje de los estudiantes, pero no es un factor decisivo en el contexto global del proceso académico en función de los diferentes instrumentos de evaluación.

En el postest, la incidencia del estudio del límite de una función constituye un prerrequisito para definir la derivada e integral de una función real, por lo tanto se implementa evaluaciones escritas que son calificadas sobre veinte puntos, en las evaluaciones el estudiante debe calcular la derivada de una función aplicando la definición, además debe calcular la integral impropia de determinadas funciones analizando si son o no convergentes. En la tabla 7, se presentan los resultados descriptivos de las evaluaciones.

Tabla 7

Estadísticas descriptivas de las calificaciones obtenidas en el Postest

\begin{tabular}{|l|c|c|c|c|}
\hline \multicolumn{1}{|c|}{ Grupos } & N & Media & $\begin{array}{c}\text { Desviación } \\
\text { típ. }\end{array}$ & $\begin{array}{c}\text { Error típ. } \\
\text { de la media }\end{array}$ \\
\hline G_Control & 30 & 15.93 & 0.98 & 0.17897 \\
\hline G_Experimentación & 30 & 17.53 & 0.97 & 0.17768 \\
\hline
\end{tabular}

Para la toma de decisiones se considera el nivel de confianza al $95 \%$ para el análisis de la igualdad de medias, en la tabla 8 , se presenta la Prueba de Levene para el análisis de la igualdad de varianzas y se obtiene un p-valor de 0.539 que es mayor al p-valor de 0.05 que representa el nivel de significancia, lo que indica que los conjuntos de datos siguen una distribución normal, con éste indicador se analiza la Prueba T para la igualdad de medias con la finalidad de poder evidenciar diferencias entre los promedios de las calificaciones de los grupos de estudiantes.

Se formula la hipótesis de investigación para la toma de decisión, siendo

$\mathrm{H}_{0}: \sigma_{1}^{2}=\sigma_{2}^{2}$

$\mathrm{H}_{1}: \sigma_{1}^{2} \neq \sigma_{2}^{2}$

Al contrastar las medias de los grupos y analizar el p-valor Sig. (bilateral) de 0.000 que es menor al 0.05 , la regla de decisión es rechazar $\mathrm{H}_{0}$ y de aceptar $\mathrm{H}_{1}$ lo que significa que las varianzas de las poblaciones son diferentes.

Tabla 8

Prueba T para la igualdad de medias entre grupos independientes

\begin{tabular}{|c|c|c|c|c|}
\hline \multicolumn{2}{|c|}{$\begin{array}{c}\text { Prueba de Levene } \\
\text { para la igualdad de } \\
\text { varianzas }\end{array}$} & \multicolumn{3}{|c|}{$\begin{array}{c}\text { Prueba T para la igualdad de } \\
\text { medias }\end{array}$} \\
\hline $\mathrm{F}$ & Sig. & $\mathrm{T}$ & $\mathrm{Gl}$ & $\begin{array}{c}\text { Sig. } \\
\text { (bilateral) }\end{array}$ \\
\hline 0.383 & 0.539 & -6.344 & 58 & 0.000 \\
\hline & & -6.344 & 57.997 & 0.000 \\
\hline
\end{tabular}

El resultado de Sig. (bilateral) utilizado para la verificación de la hipótesis induce a pensar que la utilización de las aplicaciones informáticas en el estudio de límites de una función real produjo aprendizajes significativos en el grupo de experimentación, en tanto que, en el grupo de control se detectó aprendizajes que son momentáneos y no perduran en el tiempo; por lo tanto, no trasciende en su formación académica.

\section{CONCLUSIONES}

La investigación establece que la utilización de las aplicaciones informáticas contribuye en mejorar el rendimiento académico de los estudiantes en el transcurso del curso, dado en los resultados de la tabla 8 .

Se establece una correlación entre la preferencia del estilo de aprendizaje de los estudiantes dado en la tabla 2, los estilos de aprendizaje propuestos por Kolb y Honey-Mundford descritos en la tabla 1 y la comparación del promedio de notas obtenidas en la aplicación de las diferentes metodologías de evaluación dada en la Fig. 5, se concluye que la utilización de las aplicaciones informáticas contribuye en alcanzar aprendizajes significados en el tema estudiando.

De la utilización de rubricas en las metodologías de evaluación se identifica que el grupo de control presenta dificultades en los parámetros relacionados a la utilización de materiales y equipos; así como en las exposiciones de la solución de un problema de la vida real relacionado con el 
tema estudiado.

De la investigación se desprende que el estilo de aprendizaje Teórico-Reflexivo se caracteriza por fortalecer sus habilidades creativas, criticas, de reflexión, análisis, diseño y originalidad, en tanto que los estudiantes con estilo de aprendizaje Activo-Pragmático, se caracterizan por fortalecer el dinamismo, la visualización de conceptos y procesos de aproximación, la construcción de conceptos particulares, la exploración y el descubrimiento en base al análisis de diferentes casos de estudio, enfatizando que no se requiere de una calculadora para realizar cálculos, sino una herramienta que permita fomentar la libertad de elección, participación y protagonismo del estudiante, la utilización de herramientas informáticas establecen retos en el personal docente al integrar modelos educativos, metodologías didácticas y las competencias tecnológicas como parte de una nueva cultura de educación.

\section{REFERENCIAS}

[1] Maldonado, E. R. R. (2015). Secuencias didácticas para la enseñanza del concepto de límite en el cálculo/Didactic Sequences for Teaching the Concept of Limit in Calculus. Revista Internacional de Aprendizaje en Ciencia, Matemáticas y Tecnología, 2(2). DOI: http://dx.doi.org/10.23913/ride.v6i12.227

[2] De Fanelli, A. M. G. (2014). Rendimiento académico y abandono universitario: Modelos, resultados y alcances de la producción académica en la Argentina. Revista Argentina de Educación Superior, (8), 9-38. https://doi.org/10.1016/S2007-2872(14)71954-7

[3] Cantarero, P. M. P. (2016). Factores relacionados con la deserción en el primer y segundo año de estudio en la carrera de Enseñanza de la Matemática de la Universidad Nacional de Costa Rica. Revista Electrónica Educare, 20(1), 1. DOI: https://doi.org/10.15359/ree.20 1.5

[4] Cejas León, R., Navío Gámez, A., \& Barroso Osuna, J. M. (2016). Las competencias del profesorado universitario desde el modelo TPACK (conocimiento tecnológico y pedagógico del contenido). Pixel-Bit. Revista de Medios y Educación, 49, 105-119.

[5] González, F. E. (2016). Los nuevos roles del profesor de matemática Retos de la Formación de Docentes para el Siglo XXI. Paradigma, 21(1), 139-172.

[6] Cheung, A. C., \& Slavin, R. E. (2013). The effectiveness of educational technology applications for enhancing mathematics achievement in K12 classrooms: A meta-analysis. Educational research review, 9, 88113. https://doi.org/10.1016/j.edurev.2013.01.001

[7] Gravemeijer, K., Stephan, M., Julie, C., Lin, F. L., \& Ohtani, M. (2017). What Mathematics Education May Prepare Students for the Society of the Future? International Journal of Science and Mathematics Education, 15(1), 105-123. https://doi.org/10.1007/s10763-017-9814-6

[8] Niss, M., Bruder, R., Planas, N., Turner, R., \& Villa-Ochoa, J. A. (2016). Survey team on: conceptualisation of the role of competencies, knowing and knowledge in mathematics education research. Zdm, 48(5), 611-632. https://doi.org/10.1007/s11858-016-0799-3

[9] Vargas, W. M. R., Layedra, N. D. P. B., \& Pilco, J. M. O. (2018). Software libre matemático y su incidencia en el aprendizaje del cálculo diferencial. mktDESCUBRE, 1(Décima), 102-110.

[10] Tall, D., \& Katz, M. (2014). A cognitive analysis of Cauchy's conceptions of function, continuity, limit and infinitesimal, with implications for teaching the calculus. Educational Studies in Mathematics, 86(1), 97-124. https://doi.org/10.1007/s10649-014 9531-9

[11] Aguilar Juárez, I., Ayala De la Vega, J., Lugo Espinosa, O., \& Zarco Hidalgo, A. (2014). Análisis de criterios de evaluación para la calidad de los materiales didácticos digitales. Revista iberoamericana de ciencia tecnología y sociedad, 9(25), 73-89.

[12] Díaz-Ovalle, C. O., Rico, A. K., Arellano, A., \& Guzmán-Zazueta, A (2013). Estrategia para detectar Estilos de Aprendizaje usando la técnica de particiones. Journal of Learning Styles, 6(12).

[13] Cué, J. L. G., Rincón, J. A. S., \& García, C. M. A. (2009). Instrumentos de medición de estilos de aprendizaje. Journal of Learning Styles, 2(4)
[14] Herran, A. O., Marín, P. A. R., \& Méndez, N. D. D. (2018). Recomendación de Estrategias de Aprendizaje Personalizadas Basadas en el Test de CHAEA. Scientia et technica, 23(2), 222-229.

[15] Bautista, J. E. C. (2006). Identificación de los estilos de aprendizaje en los estudiantes de fisiología del ejercicio de la Facultad de Rehabilitación y Desarrollo Humano. Revista ciencias de la salud, 4.

[16] Godino, J. D. (2014). Indicadores de idoneidad didáctica de procesos de enseñanza y aprendizaje de las matemáticas.

[17] Aguado, M. L., \& Falchetti, E. S. (2009). Estilos de aprendizaje. Relación con motivación y estrategias. Journal of Learning Styles, 2(4).

[18] López, C., Benedito, V., \& León, M. J. (2016). El Enfoque de Competencias en la Formación Universitaria y su Impacto en la Evaluación: La Perspectiva de un Grupo de Profesionales Expertos en Pedagogía. Formación universitaria, 9(4), 11-22.

[19] Lugo, C. S. J., Hernandez, G. R., \& Montijo, E. L. (2012). El cuestionario de estilos de aprendizaje CHAEA y la escala de estrategias de aprendizaje ACRA como herramienta potencial para la tutoría académica. Journal of Learning Styles, 5(10).

[20] Escanero-Marcén, J. F., Soria, M. S., Escanero-Ereza, M. E., \& GuerraSánchez, M. (2013). Influencia de los estilos de aprendizaje y la metacognición en el rendimiento académico de los estudiantes de fisiología. FEM: Revista de la Fundación Educación Médica, 16(1), 23 29.

[21] Arbain, N., \& Shukor, N. A. (2015). The effects of GeoGebra on students achievement. Procedia-Social and Behavioral Sciences, 172, 208-214. https://doi.org/10.1016/j.sbspro.2015.01.356

[22] Laal, M., \& Ghodsi, S. M. (2012). Benefits of collaborative learning. Procedia-social and behavioral sciences, 31, 486-490. https://doi.org/10.1016/j.sbspro.2011.12.091

[23] Vergnaud, G. (2016). ¿ En qué sentido la teoría de los campos conceptuales puede ayudarnos para facilitar aprendizaje significativo? Investigações em ensino de ciências, 12(2), 285-302.

[24] Zengin, Y., Furkan, H., \& Kutluca, T. (2012). The effect of dynamic mathematics software geogebra on student achievement in teaching of trigonometry. Procedia-Social and Behavioral Sciences, 31, 183-187. https://doi.org/10.1016/j.sbspro.2011.12.038

[25] Díaz-Antón, G., Pérez, M., Grimán, A., \& Mendoza, L. (2002). Instrumento de evaluación de software educativo bajo un enfoque sistémico. Recuperado el, 6.

[26] Rodríguez, M. (2011). Exploracion del impacto producido por la integración del ambiente de geometría dinámica (AGD) geogebra en la enseñanza de los cursos de matemáticas básicas de primer semestre de la Universidad Nacional de Colombia sede Palmira. National University of Colombia.

[27] Aqda, M. F., Hamidi, F., \& Rahimi, M. (2011). The comparative effect of computer-aided instruction and traditional teaching on student's creativity in math classes. Procedia Computer Science, 3, 266-270.

[28] Vogel, F., Kollar, I., Ufer, S., Reichersdorfer, E., Reiss, K., \& Fischer, F. (2016). Developing argumentation skills in mathematics through computer-supported collaborative learning: The role of transactivity. Instructional Science, 44(5), 477-500. 